\title{
Coloração de biopolímero biodegradável utilizando-se corantes naturais betanina e curcumina
}

\section{Biodegradable biopolymer coloring using betanin and curcumine natural dyes}

\author{
1 Pamella Carvalho Gonçalves pamellacargon@icloud.com \\ 1 Renata Carolina Zanetti Lofrano
}

1 Universidade Federal de São João Del Rei/UFSJ

\section{Resumo}

O uso de matérias-primas derivadas de fontes naturais tem sido cada vez mais estudado e empregada na produção dos mais diversos tipos de materiais, dentre eles, os biopolímeros. 0 estudo aqui relatado apresenta os resultados preliminares da coloração do biopolímero biodegradável poli(citrato de glicerol) com os corantes naturais e comerciais, curcumina e betanina. $\mathrm{Na}$ coloração, foram empregados $10,0 \mathrm{~g}$ do biopolímero e $0,005 \mathrm{~mol}$ do corante pulverizado. Os biopolímeros coloridos foram, então, submetidos a ensaios de secagem em estufa, em dessecador, em chapa de aquecimento, em diferentes temperaturas, em atmosfera ambiente e sob vácuo, com e sem exposição à luz natural, no decorrer de duas semanas. Os resultados obtidos demonstram que o biopolímeros foram coloridos satisfatoriamente empregando-se ambos os corantes, nas condições estudadas e mostraram-se visualmente estáveis ao aquecimento, sob vácuo e atmosfera e luz ambientes no período de tempo avaliado.

\section{Palavras-chave:}

Biopolímero biodegradável. Corantes naturais. Betanina. Curcumina.

\begin{abstract}
The use of raw materials derived from natural sources has been increasingly studied and employed in the production of the most diverse types of materials, among them biopolymers. The study reported here presents the preliminary results of the staining of biodegradable poly (glycerol citrate) biopolymer with the natural and commercial dyes, curcumin and betanin. In the staining, $10.0 \mathrm{~g}$ of the biopolymer and $0.005 \mathrm{~mol}$ of the powdered dye were used. The colored biopolymers were then subjected to oven drying, desiccator, heating plate, at different temperatures, under ambient and vacuum conditions, with and without exposure to natural light, within two weeks. The results obtained demonstrate that the biopolymers were satisfactorily colored using both dyes under the conditions studied and were visually stable to heating under vacuum and at ambient and ambient light conditions over the period of time evaluated.
\end{abstract}

\section{Keywords:}

Biodegradable biopolymer. Natural dyes. Betanin. Curcumin.

\section{Como você deve citar?}

GONÇALVES, Pamella Carvalho; LOFRANO, Renata Carolina Zanetti. Coloração de biopolímero biodegradável utilizando-se corantes naturais betanina e curcumina. Cadernos UniFOA, Volta Redonda, n. 38, p. 51-56, dez. 2018. 
Coloração de biopolímero biodegradável utilizando-se

corantes naturais betanina e curcumina

\section{INTRODUÇÃO}

A realização de inúmeras pesquisas, em diferentes áreas do conhecimento, permitiu desenvolvimento de novas tecnologias e materiais empregando-se insumos provenientes de fontes renováveis, tais como, os biopolímeros. Suas propriedades como biodegradabilidade, biocompatibilidade, atividades antimicrobiana e/ou antioxidante e baixa toxicidade impulsionaram estudos, visando-se diversas aplicações, tais como, em têxteis, embalagens, próteses, adesivos, membranas de filtração e outros [1-3]. Alguns desses usos estão atrelados à disponibilidade dos polímeros em uma extensa variedade de cores e, para tal, são adotados corantes e pigmentos orgânicos e inorgânicos naturais $[4,5]$.

Corantes são compostos químicos (ou misturas) adicionados a materiais ou produtos, tais como alimentos, bebidas, polímeros e outros, com a finalidade de lhes conferir ou intensificar sua coloração [6]. Mais recentemente, os corantes orgânicos naturais, de origem vegetal ou animal, tais como extratos de urucum, carmim cochonilha, curcumina, antocianinas e betalaínas têm sido citados em vários estudos e pesquisas $[7,8]$.

Vários corantes naturais conferem cor, mas também possuem propriedades benéficas à saúde humana, devido às suas características antioxidantes e anti-inflamatórias. Dessa forma, o seu uso torna-se conveniente e interessante, pois melhoram ou modificam o aspecto visual do produto, além de promover benefícios à saúde de quem os consome. Outro fator que impulsionou o uso de compostos ou produtos naturais foi à conscientização ecológica da população, que tornou imperativo o emprego industrial desses corantes em alimentos, medicamentos, cosméticos e etc [9].

A curcumina é proveniente do tubérculo Turmeric ou Curcuma longa. Apresenta uma cor amarelo-esverdeado em solução e é utilizada em cosméticos, medicamentos, têxteis e alimentos em função de suas atividades farmacológicas anti-inflamatórias, antiviral, antibactericida, antioxidante, antifúngica, anticarcinogênica, entre outras ações terapêuticas [10,11].

A beterraba é a principal fonte de betalaínas, compostos solúveis em água, responsáveis pela coloração vermelha, amarela, rosa e laranja em flores e frutas. Podem ser divididas em betacianinas (vermelho ao vermelho violeta) e betaxantinas (amarelo). As betacianinas são classificadas, em função de sua estrutura química, em betanina, amarantina, gonferina e bougainvilina [12]. As betaninas são pigmentos encontrados exclusivamente em plantas, como a Beta vulgaris (beterraba). Constituem uma excelente fonte de pigmentos vermelhos $[13,14]$.

Dessa forma, o estudo preliminar aqui apresentado, teve por objetivo colorir o biopolímero poli(citrato de glicerol), com os corantes naturais curcumina e betanina comerciais, e avaliar suas estabilidades ao aquecimento, atmosfera e luz ambientes no decorrer do tempo. Tem-se, assim, o desenvolvimento de um biopolímero colorido, biodegradável e ecologicamente adequado, produzido principalmente a partir de matérias primas renováveis e não tóxicas, tais como o ácido cítrico, curcumina e betanina.

\section{MATERIAIS E MÉTODOS}

\subsection{Materiais}

Materiais: Ácido cítrico (98\%, Synth); glicerol (99\%, Sigma-Aldrich); betanina (Beterraba E162 Colores Naturales); curcumina (Curcumina E100 - Colores Naturales). 
Equipamentos: Balança semi-analítica (Marte AY220); Chapa aquecedora/agitadora (LS LOGEN); estufa de secagem e esterilização (SLABMA 1351100); banho-maria (Novatécnica NT 268)

\subsection{Métodos}

O biopolímero foi preparado, conforme descrito por De Rezende e Lofrano (2014) [15]. Em seguida, $10,0 \mathrm{~g}$ do biopolímero a, aproximadamente, $25^{\circ} \mathrm{C}$, foram transferidas para um béquer de $100 \mathrm{~mL}$, e adicionados $0,005 \mathrm{~mol}$ do corante pulverizado betanina ou curcumina. Após sua homogeneização por agitação magnética, a mistura foi disposta em um vidro relógio e levado à estufa durante $24 \mathrm{~h}$ para completa secagem do material. A secagem foi realizada nas temperaturas de 75,85 e $95^{\circ} \mathrm{C}$. Foram realizados também estudos de secagem em dessecador, à temperatura ambiente e sob vácuo, sem e sob a exposição à luz natural, durante duas semanas.

\section{RESULTADOS E DISCUSSÃO}

Biopolímeros podem ser definidos como polímeros (proteínas, ácidos nucleicos, polissacarídeos) produzidos por organismos vivos ou a partir de compostos oriundos de fontes renováveis, tais como carboidratos, presentes na cana-de-açúcar, milho, batata, trigo e beterraba; ou um óleo vegetal extraído de soja, girassol, palma ou de outra planta oleaginosa [4]. Dentre eles, recentemente, destacam-se o poli(ácido glicólico) (PGA), poli(ácido lático) (PLA), poli( $\varepsilon$-caprolactona) e o e poli(ácido /-lático-co-glicólico) (PLGA) e combinando-se o ácido cítrico e/ou glicerol a outros compostos, por exemplo, como no caso do poli(sebacato de glicerol), poli(citrato de octanodiol) e poli(citrato co-sebacato de octanodiol) [16].

Os estudos realizados objetivaram identificar as condições adequadas de coloração do biopolímero biodegradável poli(citrato de glicerol), nas quais fossem mantidas as cores originais, de forma homogênea e estável dos corantes betanina e curcumina, evitando-se a degradação ou coloração irregular do biopolímero. Esses corantes foram escolhidos devido à sua disponibilidade comercial, custo pouco oneroso, não toxicidade e propriedades antibacteriana e antioxidante [14, 17], tornando possível a obtenção de biopolímeros coloridos com as mesmas propriedades dos corantes adotados.

Quando a adição dos corantes foi realizada à temperatura ambiente, verificou-se que, após a homogeneização das misturas, as cores vinho e amarela características dos corantes betanina e curcumina, respectivamente, foram preservadas e se mantiveram estáveis e uniformes (Figura 1 (A) e (B)). 
Figura 1 - Imagens dos biopolímeros coloridos, à temperatura ambiente, com betanina (A) e com curcumina (B); mantidos $1 \mathrm{~h}$ de aquecimento a $115^{\circ} \mathrm{C}$ com curcumina (C) e com betanina (D); após 1 semana de secagem em dessecador, sob vácuo e à temperatura ambiente com e sem proteção à luz ambiente, com curcumina (E) e (F) e com betanina $(G)$ e $(H)$, respectivamente.

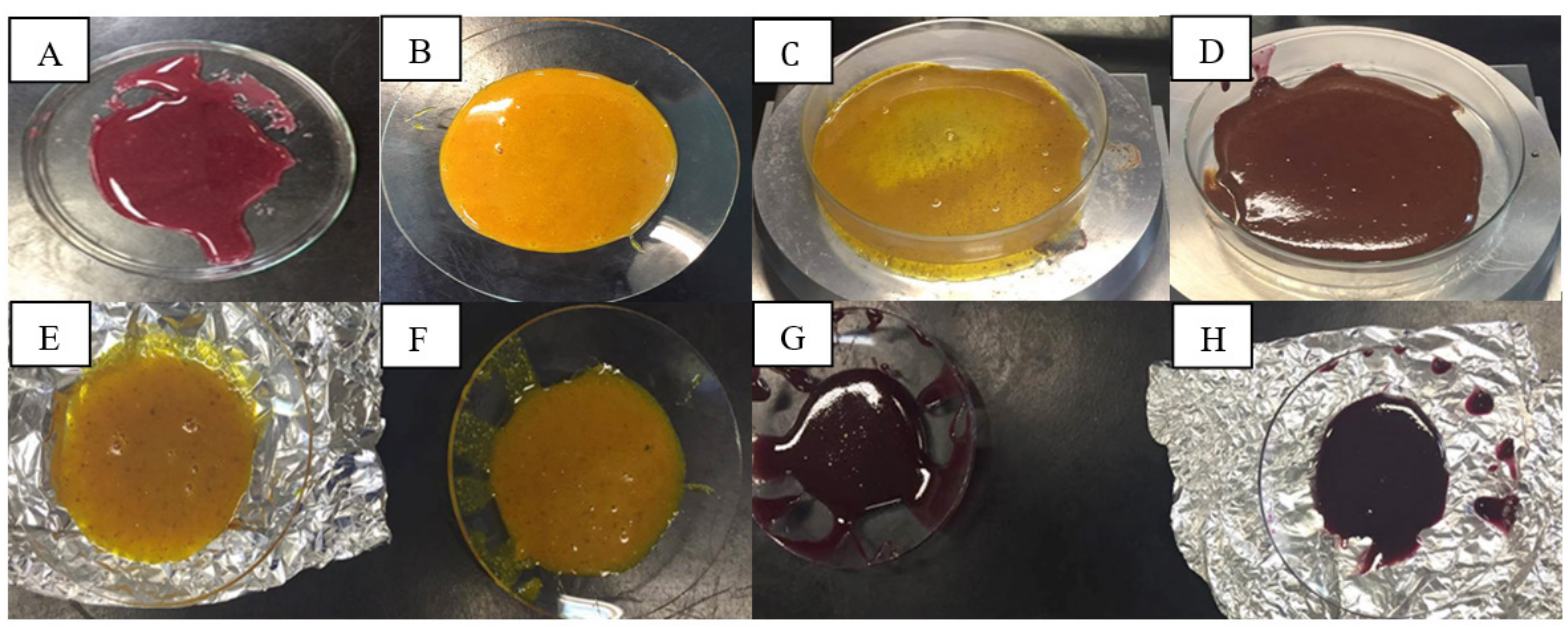

Fonte: dos autores, 2018.

Quando os biopolímeros coloridos foram submetidos à temperatura de $115^{\circ} \mathrm{C}$ por $1 \mathrm{~h}$, em placa de aquecimento, verificou-se que o material colorido com a curcumina, apresentou uma coloração amarela e translucida (Figura 1 (C)). Tal resultado está de acordo com a literatura, que relata que a curcumina é extraordinariamente estável ao calor e pode se utilizada em produtos de qualquer grau de acidez [14].

No caso da betanina, o material mostrou-se turvo e de cor marrom (Figura 1 (D)), indicando que a betanina é instável à temperatura empregada $\left(115^{\circ} \mathrm{C}\right)$ e/ou à acidez do meio, devido à presença de ácido cítrico residual da polimerização do poli(citrato de glicerol), cuja taxa de conversão do ácido cítrico e glicerol em polímero foi de, aproximadamente, $78 \%$. Tal resultado inviabiliza seu uso como corante do biopolímero, sem que o mesmo tenha sido submetido a processo de purificação, para remoção do ácido cítrico que não foi consumido na polimerização. Tal instabilidade à temperatura elevada e ao pH está de acordo com os resultados observados por Volp, Renhe, Stringueta (2009), que verificaram que a estabilidade da betanina depende do $\mathrm{pH}$ (estabilidade excelente: $\mathrm{pH} 4-5$ e razoável: $\mathrm{pH} 3-7$ ) e é instável em presença de luz e oxigênio, sendo destruída, quando submetida a altas temperaturas [12].

Tais estudos foram realizados visando-se verificar a possibilidade de coloração dos biopolímeros, imediatamente após a sua polimerização, que ocorre a $115^{\circ} \mathrm{C}$, evitando-se assim, o tempo exigido para que o material atinja temperaturas mais baixas.

Nos ensaios realizados em estufa para a secagem dos biopolímeros coloridos, em diferentes temperaturas $\left(75,85\right.$ e $\left.95^{\circ} \mathrm{C}\right)$, verificou-se que o corante curcumina $\left(95^{\circ} \mathrm{C}\right)$ suporta temperaturas mais elevadas que a Betanina $\left(85^{\circ} \mathrm{C}\right)$, devido a alterações nas colorações observadas a essas temperaturas, em relação às colorações originais dos biopolímeros. Tais resultados afetarão o processo de secagem, dificultando e prolongando a remoção de água presente nos biopolímeros coloridos. Nas amostras secas em dessecador à temperatura ambiente e sob vácuo, com e sem exposição à luz natural, após duas semanas (Figura $1(E),(F),(G),(H)$ ), verificou-se que, mesmo sob a exposição à luz natural, no período de tempo estudado, houve a manutenção, homogeneidade e brilho, na coloração de ambos os biopolímeros, ou seja, os corantes betanina e curcumina não foram fotodegradados nas condições 
estudadas. Esses resultados estão de acordo com os dados apresentados na literatura quanto à estabilidade ou fotossensibilidade dos corantes estudados [14].

\section{CONCLUSÕES}

Os resultados obtidos nos permitiram concluir que os corantes curcumina e betanina foram satisfatórios e promissores na coloração do biopolímero biodegradável poli(citrato de glicerol) na diferentes condições investigadas.

\section{REFERÊNCIAS}

[1] DE AZEVÊDO, L. C.; CORDEIRO DE SÁ, A. S.; ROVANI, S.; FUNGARO, D. A. Propriedades do amido e suas aplicações em biopolímeros. Cadernos de Prospecção, vol. 11, p. 351-358, 2018.

[2] DE FARIAS, S. S.; SIQUEIRA, S. M. C.; CRISTINO, J. H. S.; DA ROCHA, J. M. Biopolímeros: uma alternativa para promoção do desenvolvimento sustentável. Revista Geonorte, vol. 7, n² 26, p. 61- 77, 2016.

[3] BORSCHIVER, S.; ALMEIDA, L. F. M.; ROITMAN, T. Monitoramento tecnológico e mercadológico de biopolímeros. Polímeros, vol. 18, n³, p.256-261, 2008.

[4] BRITO, G. F.; AGRAWAL, P.; ARAÚJO, E. M.; MÉLO, T. J. A. Biopolímeros, Polímeros Biodegradáveis e Polímeros Verdes. Revista Eletrônica de Materiais e Processos, vol. 6, n² 2, p. 127-139, 2011.

[5] PIRES, A. L. R.; BIERHALZ, A. C. K.; MORAES, A. Biomateriais: Tipos, Aplicações e Mercado. Química Nova, vol. 38, no 7, p. 957-971, 2015.

[6] MARMITT, D. J.; REMPEL, C.; GOETTERT, M. I.; DO COUTO E SILVA, A. Análise da produção científica do Curcuma longa L. (açafrão) em três bases de dados. Rev Pan-Amaz Saúde, vol. 7, nº 1, p. 71-77, 2016.

[7] NETTO, R. C. M.; Dossiê dos Corantes, Foods Ingredients Brasil, vol. 9, p. 40-45, 2009.

[8] DIAS, M. V.; GUIMARÃES, P. I. C.; MERÇON, F., Corantes naturais como indicadores de pH. Química Nova na Escola, no 17, p. 27-31, 2003.

[9] DE SOUZA, R. M. Corantes naturais alimentícios e seus benefícios à saúde. Brasil, 2012 Trabalho de Conclusão de Curso (TCC) (Graduação em Farmácia), UEZO, Centro universitário Estadual Zone Oeste, São Paulo, 2012.

[10] AMMON, H. P. T.; WAHL, M. A. Pharmacology of Curcuma longa. Planta Med., vol. 57, nº 1, p. 1-7, 1991.

[11] LEON, L. L.; ARAÚJO, C. A. C. Biological activities of Curcuma longa L. Mem Inst Oswaldo Cruz, vol. $96, n^{\circ} 5$, p. $723-728,2001$.

[12] VOLP, A. C. P.; RENHE, I. R. T.; STRINGUETA, P. C. Pigmentos Naturais Bioativos. Alim. Nutr., v. 20, no 1, p. 157-166, 2009.

[13] CUCHINSKI, A. S.; CAETANO, J.; DRAGUNSKI, D. C. Extração do corante da beterraba (beta vulgaris) para utilização como indicador ácido-base. Ecl. Quím., vol. 35, no 4, p. 17-23, 2001. 
[14] MARCOLINO, V. A. Inclusão de bixina, curcumina e betanina em ciclodextrina para aplicação na indústria de alimentos. Brasil. 2008. Tese (Doutorado em Ciências de Alimentos), UNICAMP, Universidade Estadual de Campinas, São Paulo, 2008.

[15] DE REZENDE, D. J. L. F.; LOFRANO, R. C. Z. Produção de citrato de glicerol que atuam como plastificantes verdes usando-se glicerina, efluente da produção de biodiesel por transesterificação. Anais do XX Congresso Brasileiro de Engenharia Química/COBEQ - Florianópolis, SC, 2014.

[16] ORTEGA-TORO, R.; COLLAZO BIGLIARDI, S.; TALENS, P.; CHIRALT, A. Influence of citric acid on the properties and stability of starch-polycaprolactone baseds films. Journal Applied Polymer Science, vol.133, n² 2, p. 42220 (1-16), 2016.

[17] GARCÍA-CRUZ, L.; SALINAS-MORENO, Y.; VALLE-GUADARRAMA, S. Betalaínas, compuestos fenólicos y actividad antioxidante en pitaya de mayo (Stenocereus griseus $H$.) Rev. Fitotec. Mex., vol. 35, n 5, p. $1-5,2012$. 